\title{
Les paysages postmodernes de l'énergie en Rhénanie-Palatinat
}

Postmodern energy landscapes in Rhineland-Palatinate

Postmoderne Energielandschaften in Rheinland-Pfalz

Thomas Brühne, Michael Tempel and Michel Deshaies

\section{(2) OpenEdition}

\section{Journals}

Electronic version

URL: http://journals.openedition.org/rge/5426

ISSN: $2108-6478$

\section{Publisher}

Association des géographes de l'Est

Printed version

Date of publication: 1 January 2015

ISSN: 0035-3213

\section{Electronic reference}

Thomas Brühne, Michael Tempel and Michel Deshaies, « Les paysages postmodernes de l'énergie en Rhénanie-Palatinat », Revue Géographique de l'Est [Online], vol. 55 / n¹-2 | 2015, Online since 09 June 2015, connection on 08 September 2020. URL : http://journals.openedition.org/rge/5426

This text was automatically generated on 8 September 2020

Tous droits réservés 


\title{
Les paysages postmodernes de l'énergie en Rhénanie-Palatinat
}

\author{
Postmodern energy landscapes in Rhineland-Palatinate \\ Postmoderne Energielandschaften in Rheinland-Pfalz
}

Thomas Brühne, Michael Tempel and Michel Deshaies

\section{Introduction}

$1 \mathrm{Au}$ cours des dernières décennies, le développement d'unités de production énergétique décentralisée en Allemagne a changé certains paysages et produit des «paysages de l'énergie post-modernes». Tout comme les principaux éléments spatiaux de ces nouveaux paysages énergétiques, l'utilisation de sources d'énergie renouvelable a de nombreux effets sur la société et l'environnement. L'article présente l'exemple du nouveau paysage énergétique en Rhénanie-Palatinat.

La catastrophe nucléaire de Fukushima en mars 2011 a conduit l'Allemagne à remettre en cause l'utilisation civile de l'énergie nucléaire. Le moratoire de trois mois sur le nucléaire décidé à la suite des événements du Japon, ainsi que les rapports de deux comités d'experts ont finalement amené le Bundestag, le 6 Juin 2011, à approuver la proposition de treizième amendement à la loi sur l'énergie atomique (13 AtGÄndG). Depuis son entrée en vigueur le 6 Août 2011, il supprime les quotas supplémentaires de production d'électricité accordés par le onzième amendement à la loi sur l'énergie atomique (11 AtGÄndG ) et il confirme la fermeture des sept plus anciennes centrales nucléaires, ainsi que celle de la centrale de Krümmel. De plus, il prévoit une fermeture programmée des autres centrales nucléaires d'ici à l'an 2022.

Une conséquence directe de ces décisions est que dans de nombreux Länder, la politique énergétique est maintenant en discussion sous la rubrique de «transition énergétique» (Energiewende). Avec la sortie du nucléaire, on espère jusqu'au niveau des plus petites unités administratives, pouvoir bénéficier à l'avenir d'une plus grande part de la valeur ajoutée du secteur de l'énergie. 
Figure.1: Panneau indicateur du « paysage de l'énergie » de Morbach dans le Hunsrück (Photo: T. Brühne)

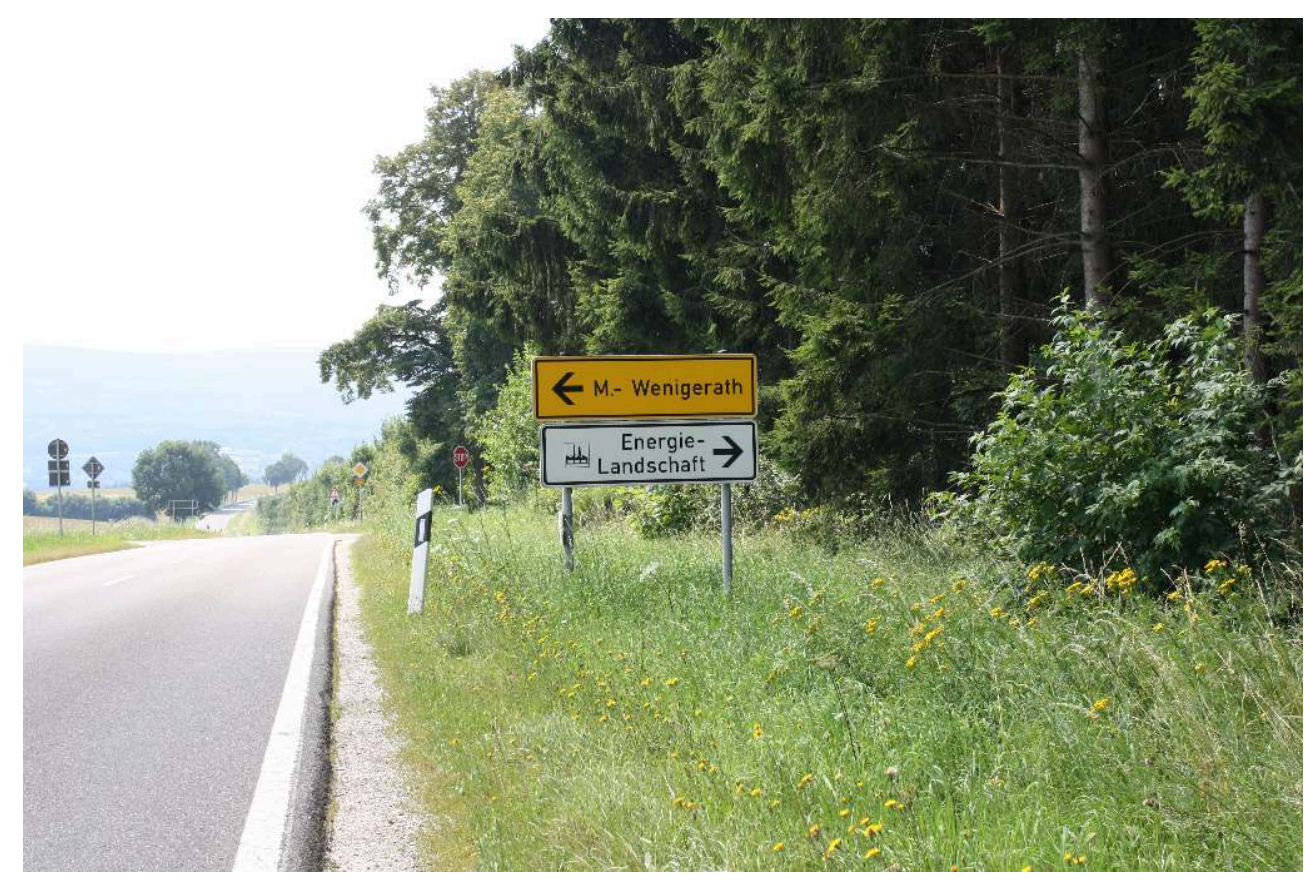

Dans le cadre du changement de paradigme de la politique énergétique en RhénaniePalatinat, les objectifs du gouvernement du Land sont particulièrement ambitieux, de telle sorte que la transition énergétique peut être considérée comme décidée. Sous les auspices du ministre de l'Énergie, Eveline Lemke, il est prévu de quintupler la production d'électricité éolienne d'ici à 2020, de faire passer la capacité actuelle des systèmes de production photovoltaïque à deux térawattheures (milliards de kWh) et d'ici à 2030, de couvrir l'ensemble des besoins en électricité à partir de sources d'énergie renouvelable (voir Figure 2). Rien que pour la production d'énergie éolienne, il est prévu selon la dernière version du Programme de développement du Land ( LEP IV), d'utiliser environ $2 \%$ de la surface du Land et au moins $2 \%$ des zones forestières ( MWKEL 2012). La réalisation imminente de cette transition énergétique en RhénaniePalatinat va conduire à un remodelage massif des paysages. Par conséquent, dans le cadre d'une recherche intégrée sur la société et l'environnement, il convient d'analyser les interventions sur les paysages naturels et les éventuels conflits que recouvre le terme de paysage énergétique post-moderne. 
Figure 2: Représentation schématique de la transition énergétique prévue en Rhénanie-Palatinat et de l'émergence des paysages de l'énergie post-modernes (Source: auteur)

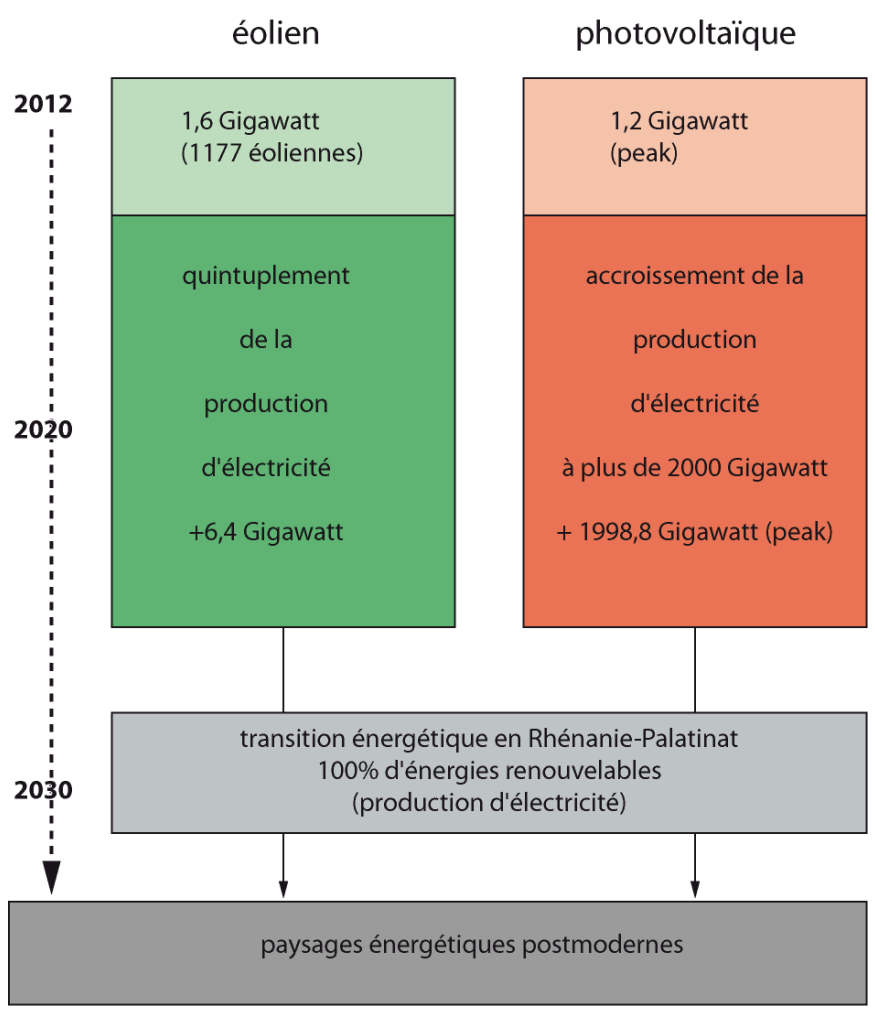

\section{Energie et paysage}

\section{A. Le concept de paysage en géographie}

Afin de caractériser le concept de paysage de l'énergie, une clarification de la notion de paysage semble inévitable. Le concept original de paysage remonte à la peinture et aux considérations esthétiques de portions de l'espace et recouvre des connotations principalement esthétiques. À partir des conceptions dérivées de l'esthétique et du romantisme, le paysage devient au cours du passage du Moyen Age aux temps modernes, un ensemble harmonieux entre l'art et la nature, en particulier dans la peinture de paysage de la Renaissance en Italie, en Allemagne et aux Pays-Bas. L'apparition du concept de paysage géographique et scientifique par contre, ne peut pas être aussi exactement définie. La littérature spécialisée fait souvent référence à une définition d'Alexander von Humboldt (1769-1859), qui avait déclaré que le paysage était le "caractère total d'une région de la Terre ", (voir, par exemple Leser, 1997). Cependant, cette référence à la notion de paysage scientifique dans les écrits de Humboldt a été systématiquement révisée par les travaux de von Hard ( 1970a , 1970b ). La conception qu'avait Humboldt du paysage peut être plutôt considérée comme étant encore très esthétique.

6 Les concepts dominants dans la bibliographie tendent à distinguer dans la conception géographique du paysage, une perspective anthropique d'une perspective physique. Selon Neef (1967, p. 36), la géographie physique considère le paysage comme «une partie concrète de la surface terrestre caractérisée par une structure uniforme et de même composition» et voit ainsi le paysage comme une partie définissable de l'espace 
terrestre à structure homogène. À partir de cette définition, Leser (1997) désigne le paysage d'un point de vue systémique comme étant le représentant spatial de l'écosystème fonctionnel sous-jacent. Par contre, selon la géographie culturelle classique ou nouvelle, le concept de paysage est considéré comme étant certes l'ensemble d'une partie de la surface terrestre ; mais on distingue cependant des paysages en tant que produits de services sociaux et culturels (voir Hartke 1959) et comme construits de la perception humaine et de l'interprétation des sens. La géographie humaine essaie donc d'attribuer au paysage un caractère socio-spatial ou discursif et d'interpréter les pratiques et les productions sociales sous forme de traces spatiales et culturelles (voir Hard 1989). Étroitement associée à la notion de paysage de la géographie humaine, la notion de paysage culturel (Kulturlandschaft), qui remonte à des considérations de géographie historique, analyse plutôt le remodelage des éléments culturels et historiques qui forment un paysage (culturel) (voir Schenk 2002). L'interprétation physique du paysage est donc dans l'ensemble plus orientée vers la notion de système, alors que le concept de paysage de la (nouvelle) géographie culturelle repose en grande partie sur des théories constructivistes. Indépendamment de l'incompatibilité théorique des deux concepts de paysage, les différentes considérations mettent plus ou moins l'accent sur la délimitation spatiale d'une portion de la terre comme plus petit dénominateur commun.

\section{B. Caractérisation des paysages de l'énergie}

7 La désignation de portions de la surface terrestre, comme indiqué ci-dessus, implique une diversité de significations du terme paysage. Dans la suite de l'article, la notion de paysage est discutée en mettant l'accent sur les sources d'énergie et leur utilisation et donc en employant le concept de paysage énergétique. Les paysages de l'énergie sont caractérisés par le fait que l'utilisation concentrée d'une ou plusieurs sources d'énergie s'exprime clairement dans le paysage et en conséquence ces sources d'énergie sont considérées comme ayant une forte influence territoriale. Ce phénomène existe et évolue suivant les différentes périodes énergétiques (voir le tableau 1).

Les premiers paysages énergétiques perceptibles en Allemagne remontent à l'ère préindustrielle, en particulier au début du Moyen Age. Les sociétés agraires de l'époque ont façonné des paysages par l'utilisation ciblée du flux d'énergie solaire. Générées par l'insolation, les énergie telles que le vent, les eaux courantes et le bois issu des forêts denses d'Europe centrale et occidentale étaient alors considérées comme les sources d'énergie des activités économiques de l'époque et elles formaient donc les premiers paysages de l'énergie. Aujourd'hui, les traces des paysages énergétiques préindustriels sont encore partiellement lisibles à travers des héritages culturels sous forme de moulins à vent ou à eau, ainsi que par des irrégularités créées par le défrichement dans la structure des forêts. Dans la période énergétique suivante, l'âge industriel, il s'agissait principalement d'une production, d'une distribution et d'une utilisation centralisée de sources d'énergie fossiles et nucléaires très concentrées qui, en raison même de leur mode de gisement, n'ont pu être extraites que dans relativement peu de régions d'Allemagne (par exemple le charbon de la Ruhr et de la Sarre, le lignite de Rhénanie et de Lusace, ainsi que le pétrole de Basse -Saxe et du Schleswig -Holstein). Les paysages de l'énergie de l'ère industrielle sont ainsi constitués principalement de quelques unités de production d'énergie, peu nombreuses, mais particulièrement marquantes et perceptibles dans le paysage. Parmi les exemples les plus frappants, on 
peut citer les paysages énergétiques du bassin de lignite rhénan qui s'est constitué au fil des décennies, avec ses surfaces réhabilitées, ainsi que les paysages du bassin charbonnier de la Ruhr.

Tableau 1: Paysages de l'énergie d'hier et d'aujourd'hui

\begin{tabular}{|c|c|c|c|}
\hline Phase & Période & $\begin{array}{l}\text { sources } \\
\text { d'énergie } \\
\text { principalement } \\
\text { utilisées }\end{array}$ & $\begin{array}{l}\text { caractérisation du paysage } \\
\text { énergétique }\end{array}$ \\
\hline \multirow{3}{*}{$\begin{array}{l}\text { Période } \\
\text { énergétique } \\
\text { préindustrielle }\end{array}$} & $\begin{array}{l}\text { de } 1 \text { Mio. à } 10.000 \\
\text { av. JC/ sociétés de } \\
\text { chasseurs et de } \\
\text { cueilleurs }\end{array}$ & Force humaine & $\begin{array}{l}100 \% \text { de végétation naturelle, unités } \\
\text { de production d'énergie non } \\
\text { perceptibles dans le paysage }\end{array}$ \\
\hline & $\begin{array}{l}\text { de } 10.000 \text { à } 5000 \text { av. } \\
\text { JC } \\
\text { sociétés agricoles } \\
\text { simples }\end{array}$ & $\begin{array}{l}\text { Force humaine } \\
\text { et animale }\end{array}$ & $\begin{array}{l}20 \% \text { de terres cultivées; unités de } \\
\text { production d'énergie non perceptibles } \\
\text { dans le paysage }\end{array}$ \\
\hline & $\begin{array}{l}\text { de } 5000 \text { av. JC à } \\
1870 \\
\text { sociétés agricoles }\end{array}$ & $\begin{array}{l}\text { Force éolienne } \\
\text { et hydraulique, } \\
\text { bois }\end{array}$ & $\begin{array}{l}\text { Nombreuses petites unités locales de } \\
\text { production d'énergie; flux d'énergie } \\
\text { solaire contrôlés; forte dépendance } \\
\text { des surfaces; unités de production } \\
\text { d'énergie pour la première fois visibles } \\
\text { dans le paysage }\end{array}$ \\
\hline $\begin{array}{l}\text { Période } \\
\text { énergétique } \\
\text { industrielle }\end{array}$ & à partir de 1870 & $\begin{array}{l}\text { Charbon, } \\
\text { pétrole, gaz } \\
\text { naturel, } \\
\text { combustible } \\
\text { nucléaire }\end{array}$ & $\begin{array}{l}\text { Unités de production d'énergie visibles } \\
\text { dans le paysage; empreinte durable } \\
\text { dans le paysage de l'extraction de } \\
\text { l'énergie }\end{array}$ \\
\hline $\begin{array}{l}\text { Période } \\
\text { énergétique } \\
\text { postindustrielle }\end{array}$ & & $\begin{array}{l}\text { Energie } \\
\text { éolienne, force } \\
\text { hydraulique, } \\
\text { énergie solaire, } \\
\text { géothermie, } \\
\text { biomasse }\end{array}$ & $\begin{array}{l}\text { Nombreuses petites unités locales de } \\
\text { production d'énergie; flux d'énergie } \\
\text { solaire contrôlée; forte dépendance } \\
\text { des surfaces; unités de production } \\
\text { d'énergie fortement visibles dans le } \\
\text { paysage }\end{array}$ \\
\hline
\end{tabular}

D’après Sieferle (1987), Brücher (2008)

9 Dans la caractérisation des paysages de l'énergie post-modernes en tant que portions émergentes de la surface terrestre de l'ère post-industrielle, il se pose la question de leur délimitation spatiale et de la transition vers d'autres paysages. Les paysages postindustriels de l'énergie sont généralement dominés par une source d'énergie renouvelable, complétée par d'autres sources d'énergie. Celles-ci impriment leur marque sur une portion de la surface de la Terre; ce qui entraîne un effet de structure complexe entre l'homme et l'environnement. Le paysage postmoderne de l'énergie est basé sur une utilisation très innovante de l'énergie solaire et est caractérisé (par rapport à l'ère pré-industrielle) par une forte dépendance de la surface. Les potentiels et les contraintes spatiales et naturelles déterminent le degré d'utilisation des différentes sources d'énergie et influencent ainsi l'apparence de ces paysages postmodernes de l'énergie. Les régions ventées par exemple sont à l'origine de vastes fermes éoliennes et les régions à forte insolation ont des systèmes photovoltaïques et des ensembles solaires à grande échelle.

10 L'utilisation de grandes quantités d'une ou plusieurs sources d'énergie dans une région de la terre est une condition préalable et essentielle à la genèse des paysages énergétiques post-modernes. Les unités de production énergétiques ainsi réalisées deviennent des composantes marquantes du paysage et conduisent à la définition d'une nouvelle image du paysage. Si les nouvelles relations entre les unités de production énergétiques et les éléments du paysage finissent par devenir dominantes dans l'image du paysage existant, celui-ci peut être vu comme un paysage énergétique post-moderne. Dans la suite de l'article, on va analyser des régions de concentration de 
sources d'énergie en Rhénanie-Palatinat, qui peuvent être traitées comme des paysages de l'énergie dont on va dresser une typologie.

\section{Les paysages de l'énergie en Rhénanie-Palatinat}

\section{A. Localisation des paysages de l'énergie}

11 Sur une superficie de près de 20.000 kilomètres carrés, la Rhénanie-Palatinat couvre un spectre varié de zones naturelles. Il y a au total 154 grandes unités spatiales naturelles (de quatrième ordre), qui sont regroupées en 16 principaux ensembles de niveau supérieur (voir Meynen et Schmithüsen 1953-1962). La superficie de ces grandes unités du Land de Rhénanie-Palatinat est en moyenne de $386 \mathrm{~km}^{2}$ et varie de seulement 0,08 $\mathrm{km}^{2}$ pour la plus petite (Oberagger et Wiehlbergland) à $1556 \mathrm{~km}^{2}$ pour la plus grande (Nordpfälzer Bergland). Comme le montre la figure 3, des aires naturelles entières offrent un potentiel particulier pour les sources d'énergie renouvelables. Cela se reflète aussi bien dans le nombre absolu de sources d'énergie renouvelables localisées dans certaines zones naturelles que dans la production totale d'énergie et sa composante sectorielle. Alors que les sources d'énergie renouvelables, mesurées à l'aune de leur puissance nominale, ne jouent presque aucun rôle dans certaines zones naturelles telles que le Soonwald, elles sont nettement plus présentes dans le Hunsrück, le bassin du Rhin moyen, l'Islek et l'ösling, le Bitburger Gutland, les collines de la Hesse rhénane ainsi que le Nordpfälzer Bergland. En prenant en compte la puissance nominale par rapport à la superficie, des unités naturelles telles que la forêt de Münstereifel et le rebord nord-est de l'Eifel, ainsi que le plateau du Hunsrück-Saar-Ruwer sont aussi fortement marquées par les sources d'énergie renouvelables. Actuellement, en Rhénanie-Palatinat, la production d'énergie est dominée par l'éolien, suivie par l'énergie hydraulique, la biomasse et le photovoltaïque, alors que la géothermie ne joue qu'un rôle secondaire.

12 Il existe des contrastes spatiaux importants de la répartition des sources d'énergie en fonction des unités géomorphologiques. Alors que l'énergie éolienne qui est présente dans une grande variété d'espaces naturels, est de loin la source d'énergie renouvelable la plus importante (dans certaines régions comme par exemple la forêt de Münstereifel et le nord-est du rebord de l'Eifel, elle représente même près de $100 \%$ de l'énergie produite), l'énergie hydraulique occupe une position centrale dans les vallées de la Moselle et de la Lahn, ainsi que dans le bassin du Rhin moyen. La biomasse et le photovoltaïque sont souvent présents en complément dans les différents espaces naturels. Il n'y a que dans quelques régions telles que la plaine du Rhin à IngelheimMayence, la dépression de Wittlich ou le Pfälzerwald, que l'on observe une prédominance d'une combinaison de la biomasse et du photovoltaïque. Dans l'ensemble, il existe une corrélation évidente entre les sources d'énergie renouvelables et les unités naturelles qui proviennent des potentiels et des contraintes spatiales. 
Figure 3: Principales unités naturelles en Rhénanie-Palatinat et sources d'énergie renouvelables correspondantes

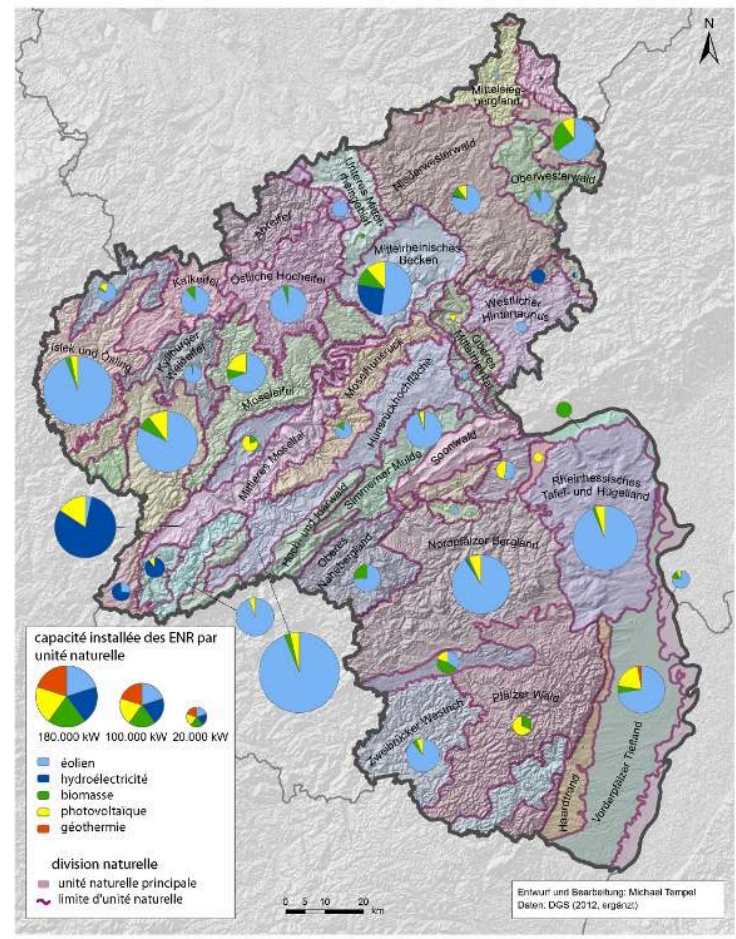

Figure 4: Paysages postmodernes de l'énergie et paysages de l'énergie en Rhénanie-Palatinat

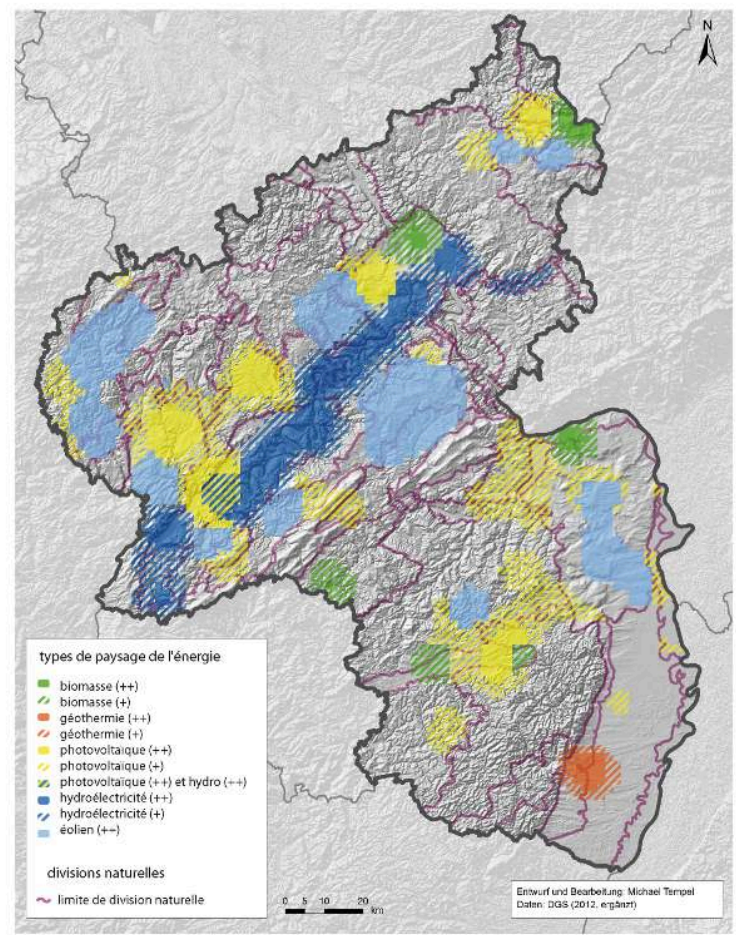

13 Cela reste un défi d'identifier les paysages énergétiques et les types de paysages de l'énergie dont les limites sont indépendantes de celles des unités naturelles. La figure 4 
montre cette délimitation en Rhénanie-Palatinat. Elle repose sur une estimation des densités, du nombre et de la capacité des installations, ainsi que sur une analyse de cluster. On peut ainsi identifier dix types de paysages énergétiques dans chacun desquels une ou plusieurs sources d'énergie jouent un rôle spatial prédominant. Ces différents types recouvrent ensemble environ $40 \%$ de la superficie du Land, alors que les $60 \%$ restants ne sont pas encore affectés par les sources d'énergie renouvelables. Un examen plus attentif révèle que seules l'hydroélectricité et le photovoltaïque forment ensemble un paysage de l'énergie. Dans tous les autres cas, une seule source d'énergie domine. Cela ne veut pas dire qu'il n'y a pas d'autres sources d'énergie renouvelables; mais celles-ci ne jouent qu'un rôle relativement mineur en ce qui concerne leur influence territoriale. Dans les types de paysage de l'énergie en Rhénanie-Palatinat, ceux de l'énergie éolienne et du photovoltaïque jouent un rôle dominant. Ensemble, ils représentent environ $23 \%$ de la superficie du Land. La répartition spatiale des paysages de l'énergie en Rhénanie-Palatinat montre essentiellement que, même s'il existe un certain lien avec les espaces naturels, les paysages énergétiques ne recouvrent pas complètement les unités naturelles et ne s'arrêtent pas non plus à leurs limites. Souvent, deux ou même plusieurs paysages de l'énergie se déploient au sein d'une même unité naturelle. Seule la vallée inférieure de la Lahn coïncide presque entièrement avec un paysage énergétique marqué par l'hydroélectricité. Cette première tentative de délimitation des paysages énergétiques en Rhénanie-Palatinat nécessite une analyse plus approfondie, par exemple sur la base de facteurs territoriaux définis précisément qui, à leur tour, doivent donner lieu à des recherches approfondies.

\section{B. Le paysage de l'énergie de Morbach}

La commune associative de Morbach est située dans le district de Bernkastel- Wittlich dans le Hunsrück. Ce qui était le plus grand dépôt de munitions des Forces aériennes américaines en Europe a été restructuré. En 2001 est née l'idée de construire un parc de l'énergie sur les 146 ha de l'ancien terrain militaire. Dans les années suivantes, on a installé un ensemble de 14 éoliennes de type Vestas V -80 (Puissance: $2 \mathrm{MW}$, production annuelle : environ 45 millions de $\mathrm{kWh}$ ), deux installations photovoltaïques de plein champ de $490 \mathrm{~kW}$ et $580 \mathrm{~kW}$ de puissance, une installation photovoltaïque de toiture de $30 \mathrm{~kW}$ et une installation de biogaz de $500 \mathrm{~kW}$ de puissance électrique et de $700 \mathrm{~kW}$ thermique. Dans le " paysage énergétique " les sources renouvelables produisent chaque année plus d'énergie que les besoins des 11.000 habitants de la commune. Dans le centre de développement adjacent, on est en train de tester une installation de méthanisation pour transformer en méthane l'excédent d'électricité produit à partir de l'énergie éolienne et solaire, afin de le stocker. Le parc énergétique marque aujourd'hui le paysage de l'ancien site militaire à un point tel qu'il est communément appelé le paysage de l'énergie. Cela soulève la question de savoir à partir de quand, d'un point de vue scientifique, on peut parler d'un paysage de l'énergie pour désigner un changement paysager qui est d'abord ponctuel. 
Figure 5: Parc éolien de Morbach, dans le district du Rhin-Hunsrück - un paysage de l'énergie du futur?

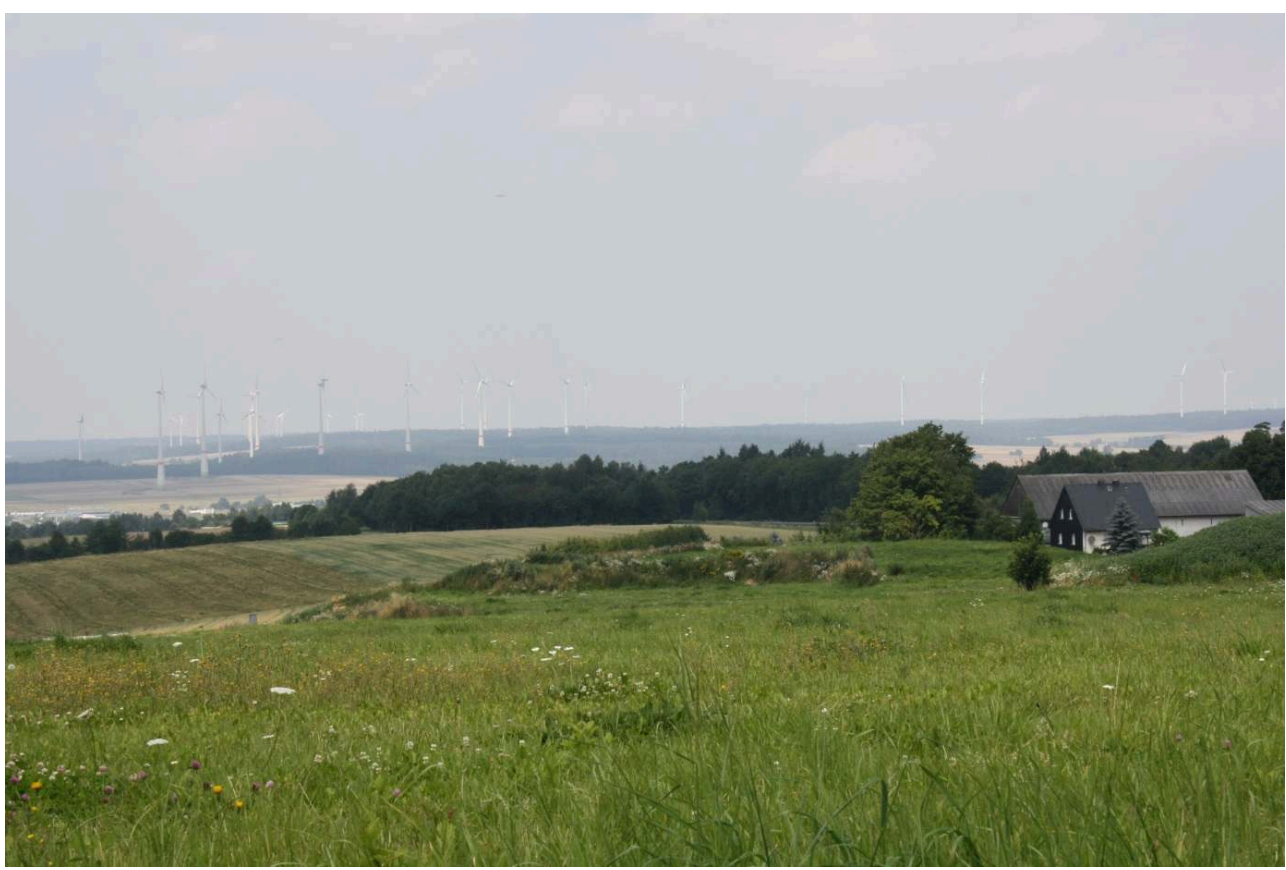

Photo: T. Brühne

\section{Les conflits potentiels entre l'homme et la nature}

En raison de l'émergence des paysages énergétiques post-modernes générés par la transition énergétique, de nouvelles zones de conflits concernant l'homme et l'environnement pourraient apparaitre dans l'avenir, ou même se renforcer (voir le tableau 2).

Tableau 2: Estimation des potentiels nouveaux de conflits au sujet des énergies renouvelables en Rhénanie-Palatinat jusqu'en 2030, à la suite de la transition énergétique

\begin{tabular}{|c|c|c|c|c|c|c|c|c|c|}
\hline & \multirow{3}{*}{ 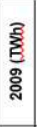 } & \multirow{3}{*}{ हूँ } & \multirow{3}{*}{ 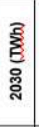 } & \multirow{3}{*}{$\begin{array}{l}\text { Mesures prioritaires pour le } \\
\text { developpement futur des } \\
\text { ENR }\end{array}$} & \multicolumn{4}{|c|}{ Effets du futur développement des ENR sur... } & \multirow{3}{*}{$\begin{array}{l}\text { Potentiel cumule } \\
\text { supplementaire } \\
\text { de conflit par le } \\
\text { développement } \\
\text { des ENR }\end{array}$} \\
\hline & & & & & \multicolumn{2}{|c|}{ Le paysage } & \multicolumn{2}{|l|}{ Le milieu naturel } & \\
\hline & & & & & $\begin{array}{c}\text { Conséquences pour le } \\
\text { paysage }\end{array}$ & $\begin{array}{c}\text { Potentiel } \\
\text { supplementaire } \\
\text { de conflit }\end{array}$ & $\begin{array}{c}\text { Conséquences pour le milieu } \\
\text { naturel (exempless) }\end{array}$ & \begin{tabular}{|c|} 
Potentiel \\
supplementaire \\
de conflit
\end{tabular} & \\
\hline \begin{tabular}{|l|}
$\begin{array}{l}\text { Force } \\
\text { bydraulique }\end{array}$ \\
\end{tabular} & 0,95 & 1,0 & 1,1 & $\begin{array}{l}\text { exclusivement par des } \\
\text { inoverations technoiques }\end{array}$ & - & - & - & & - \\
\hline Eolien & $1,68 \mid$ & 8,4 & 14,8 & $\begin{array}{l}\text { Repowering } \\
\text { construction de nouveaux } \\
\text { aécrogénérateurs }\end{array}$ & 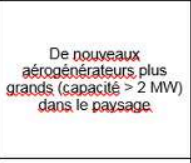 & & 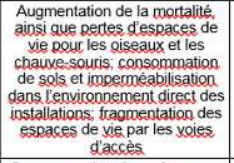 & & \\
\hline Ehotovoltaique & 0,36 & 2,0 & 5,2 & 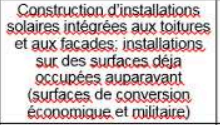 & $\begin{array}{l}\text { Installations sur des } \\
\text { surfaces visibles dans le } \\
\text { paysage: } \\
\text { changemeent optique des } \\
\text { censtructions. }\end{array}$ & & 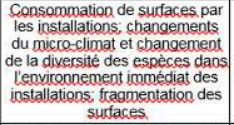 & - & \\
\hline Biomasse & 0,83 & 0,9 & 0,9 & $\begin{array}{c}\text { Cobstruction de nouvelles } \\
\text { petitis installations de biogaz } \\
(75 \mathrm{~kW})\end{array}$ & $\begin{array}{c}\text { Modification du paysage } \\
\text { bumanaise avece la culturca a } \\
\text { grande échelle de plantes } \\
\text { energétiques }\end{array}$ & - & 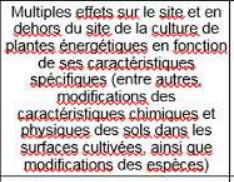 & - & 0 \\
\hline géthermie. & $0,01 \mid$ & 0,1 & 0,1 & 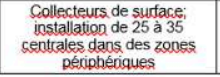 & $\begin{array}{c}\text { Centrales géothermiques } \\
\text { de taille movenenee dans le } \\
\text { paysage- }\end{array}$ & - & \begin{tabular}{|l|}
$\begin{array}{l}\text { Ehénomènes sismiques avec } \\
\text { des consegunecs poncipalement } \\
\text { sur les infrastructures bumaines }\end{array}$ \\
\end{tabular} & - & \\
\hline
\end{tabular}




\section{A. Le potentiel conflictuel de l'hydroélectricité} centrales sont souvent perçues comme ayant un effet esthétisant et romantique. De nombreuses centrales hydroélectriques telles que la centrale de style Art Nouveau de Heimbach ont été classées au patrimoine industriel après 50 ans d'activité et sont considérées comme des témoignages contemporains de l'histoire de l'énergie, bien que leur impact sur l'écosystème va durer des centaines d'années.

\section{B. Le potentiel conflictuel du photovoltaïque}

17 Après l'énergie éolienne, le photovoltaïque représente le deuxième potentiel le plus élevé de conflit. Du fait même de leur extension horizontale, les installations de plein champ apparaissent rarement dans le champ de vision des personnes, tandis que les installations de toiture sont considérées, à quelques exceptions près, comme des utilisations combinées plutôt rationnelles. Pourtant, l'intégration du photovoltaïque de façade pourrait conduire à des problèmes d'acceptation s'il se développait à grande échelle en ville. Le potentiel de conflit écologique pour cette source d'énergie peut être considéré comme modéré, étant donné que la grande extension des surfaces s'est faite sur des sites déjà fortement transformés par des usages antérieurs.

\section{Le potentiel conflictuel de l'énergie éolienne}

L'énergie éolienne présente actuellement le plus grand potentiel cumulé de conflits. Le développement de plus en plus important des éoliennes met les gens face à de nouveaux éléments paysagers qui leur sont inhabituels, avec des effets d'accoutumance probablement de plus de 50 ans (similaires à ceux concernant depuis les années 1920 et jusqu'à aujourd'hui, les lignes électriques). À une époque où les technologies de communication sont plus en plus importantes, les processus discursifs qui accompagnent les mass médias aboutissent à renforcer le potentiel conflictuel de cette source d'énergie. Le potentiel de conflit écologique de l'énergie éolienne n'est pas non plus négligeable. D'une part, il s'agit d'une altération de l'habitat des oiseaux et des chauves-souris, d'autre part la construction d'éoliennes aboutit à une imperméabilisation des sols et à un déboisement. Avec la construction de routes d'accès, il y a de plus un risque de fragmentation des habitats.

\section{Le potentiel conflictuel de la biomasse}

19 Le potentiel conflictuel de l'utilisation énergétique de la biomasse est relativement mineur étant donné son impact limité sur le paysage. En dépit des hauteurs légèrement différentes des cultures énergétiques telles que le maïs, le chanvre et le miscanthus, il existe déjà un certain degré d'acceptation. Dans ce contexte, les installations de biogaz sont cependant moins acceptables. En particulier les petites installations de production de biogaz associées à des émissions olfactives fortes, ainsi que les monocultures de plantes énergétiques exotiques pourraient encore accroître le potentiel conflictuel de cette source d'énergie. D'un point de vue écologique, la culture de plantes énergétiques variées a des effets à la fois sur place et extérieurs. Celles-ci dépendent dans le détail 
des spécificités de la culture, si bien qu'il n'est guère possible d'en tirer des considérations générales. Une gestion adéquate peut bien également entraîner des effets positifs par rapport à l'état actuel. Globalement, le potentiel futur cumulé de conflits demeure cependant relativement faible en raison des faibles taux de croissance attendus en Rhénanie -Palatinat.

\section{E. Le potentiel conflictuel de l'énergie géothermique}

20 En ce qui concerne les conflits potentiels, l'énergie géothermique se situe dans une position particulière. D'une part, l'utilisation de l'énergie géothermique est spatialement très limitée et par rapport aux autres sources d'énergie renouvelables elle occupera à l'avenir sans doute peu d'espace. D'autre part, la perception du risque de conflit est justement considérable en raison de la couverture médiatique. Le potentiel de conflits futurs attendus est limité au niveau de la perception du paysage, en lien avec la nouvelle construction de centrales entrainant des changements dans le paysage existant. Beaucoup plus important pourrait être l'impact potentiel de l'activité sismique en lien avec l'utilisation de l'énergie géothermique.

\section{Conclusion}

Le recoupement des conflits potentiels relevant des interactions entre société et cadre physique conduisent à des questions d'intégration pour la recherche sur les relations société - environnement. Dans la mesure où la politique de transition énergétique suivie actuellement par le gouvernement du Land de Rhénanie-Palatinat sera maintenue au-delà de la période législative actuelle, de nombreux nouveaux paysages énergétiques devraient émerger d'ici à 2030. Pour l'acceptation sociale de ces paysages de l'énergie se pose plus que jamais la question de savoir si l'image (construite) du paysage est toujours déterminée par son utilisation, ou si le type d'aménagement paysager et d'utilisation des sols peut être défini suivant une forme culturelle prédéterminée. De plus, la question reste ouverte de savoir si les sources d'énergie supplémentaires constituant des éléments d'un paysage changeant périodiquement vont faire l'objet d'un consensus social afin de réduire le potentiel de conflit. Les effets du développement des paysage post-modernes de l'énergie vont certainement conduire à un dilemme social : d'une part, on voudrait favoriser un niveau élevé de protection de l'environnement en limitant et en réduisant les émissions de gaz à effet de serre ; d'autre part, la forte dépendance des paysages postmodernes de l'énergie vis-à-vis de la disponibilité en surfaces entraîne par là-même des dégradations du cadre naturel.

\section{BIBLIOGRAPHY}

Braun S. \& Ziegler S., 2006, Windlandschaft. Neue Landschaften mit Windenergieanlagen. Berlin 
Brücher W., 2008, "Erneuerbare Energien in der globalen Versorgung aus historischgeographischer Perspektive”. Geographische Rundschau, 1, p. 4-12.

Brühne T. \& Tempel M., 2013, "Postmoderne Energielandschaften in Rheinland-Pfalz". Geographische Rundschau, 1, p. 28-35.

BUND [Bund für Umwelt und Naturschutz Deutschland] (Hrsg.), 2010, Energiewende in Rheinland-Pfalz. Mainz.

DGS [Deutsche Gesellschaft für Sonnenenergie e.V.] (Hrsg.), 2012, www.energymap.info/, consulté le 20.05.2012.

DRL [Deutscher Rat für Landespflege] (Hrsg.), 2006, “Die Auswirkungen erneuerbarer Energien auf Natur und Landschaft. Gutachtliche Stellungnahme und Ergebnisse des gleichnamigen Symposiums vom 19/20. Oktober 2005 in Berlin". Schriftenreihe des Deutschen Rates für Landespflege, 79 .

Hard G., 1970a, Die ,Landschaft' der Sprache und die ,Landschaft' der Geographen. Semantische und forschungslogische Studien zu einigen zentralen Denkfiguren in der deutschen geographischen Literatur. Bonn (Colloquium Geographicum 11), p. 1-278.

Hard G., 1970b, “Der „Totalcharakter der Landschaft". Re-Interpretation einiger Textstellen bei Alexander von Humboldt". In: Alexander von Humboldt. Eigene und neue Wertungen der Reisen. Arbeit und Gedankenwelt. Geographische Zeitschrift. Beihefte, p. 49-73.

Hard G., 1989, “Geographie als Spurenlesen”, Zeitschrift für Wirtschaftsgeographie (33 Jg., H. 1-2), p. 2-11.

Hartke W., 1959, “Gedanken über die Bestimmung von Räumen gleichen sozialgeographischen Verhaltens“. Erdkunde, 14 Jg., H. 4, p. 257-269.

JUWI [juwi Holding AG] (Hrsg.), 2012, www.juwi.de/energiemix/referenzen/details/ morbacher_energielandschaft-2.html ; consulté le 20.06.2012.

Leser H., 1997, Landschaftsökologie. Ansatz, Modelle, Methodik, Anwendung. Stuttgart.

Meynen E. \& Schmithüsen J. (Hrsg.), 1953-1962, Handbuch der naturräumlichen Gliederung Deutschlands. Selbstverlag der Bundesanstalt für Landeskunde. Bad Godesberg.

MWKEL [Ministerium für Wirtschaft, Klimaschutz, Energie und Landesplanung] (Hrsg.), 2012, Verordnungsentwurf der Landesregierung zur Teilfortschreibung über das Landesentwicklungsprogramm (LEP IV). Mainz

Neef E., 1967, Die theoretische Grundlage der Landschaftslehre, Gotha.

Schenk W., 2002, „Landschaft“ und „Kultur-landschaft“ - „getönte“ Leitbegriffe für aktuelle Konzepte geographischer Forschung und räumlicher Planung. Petermanns Geographische Mitteilungen 146, p. 6-13.

Sieferle R. P., 1987, Energie, in: Brüggemeier, F.-J. u. T., Rommelspacher (Hrsg.): Besiegte Natur. Geschichte der Umwelt im 19. und 20. Jahrhundert. München, p. 20-41.

\section{ABSTRACTS}

The expansion of decentralized energy supply units in Germany increasingly produces so called postmodern Renewable Energy landscapes. The key element of these landscapes is renewable 
energy technology which fundamentally affects the environment as well as society. Our paper will discuss this new phenomenon by presenting a case study of Rhineland-Palatinate.

L'expansion en Allemagne des systèmes de production énergétique décentralisés est à l'origine de ce que l'on appelle des paysages de l'énergie postmodernes. Les éléments principaux de ces paysages sont les technologies des énergies renouvelables qui affectent fortement l'environnement comme la société. Notre article discutera de ce nouveau phénomène en présentant le cas de la Rhénanie-Palatinat.

Der Ausbau dezentraler Energieversorgungseinheiten hat in den letzten Jahrzehnten in Deutschland einige Landschaften verändert und "postmoderne Energielandschaften" hinterlassen. Als die wesentlichen räumlichen Elemente dieser neuen Energielandschaften hat die Nutzung erneuerbarer Energieträger vielfältige Auswirkungen auf die Gesellschaft und Umwelt. Der Beitrag setzt sich am Beispiel von Rheinland-Pfalz mit dem neuen Phänomen der Energielandschaft auseinander.

\section{INDEX}

Mots-clés: énergie renouvelable, paysages postmodernes de l'énergie, Rhénanie-Palatinat Keywords: postmodern energy landscape, renewable energy, Rhineland-Palatinate

Schlüsselwörter: erneuerbare Energie, postmoderne Energielandschaft, Rheinland-Pfalz

\section{AUTHORS}

\section{THOMAS BRÜHNE}

Universität Koblenz - bruehne@uni-koblenz.de

MICHAEL TEMPEL

Universität Koblenz - mtempel@uni-koblenz.de

MICHEL DESHAIES

Université de Lorraine - michel.deshaies@univ-lorraine.fr 